\title{
MONTE CARLO SIMULATION OF SPIN RELAXATION OF CONDUCTION ELECTRONS IN SILICON
}

\author{
D. Persano Adorno, C. Graceffa, N. Pizzolato, and M.A. Lodato \\ Department of Physics and Chemistry, Group of Interdisciplinary Physics and CNISM, University of Palermo, \\ Viale delle Scienze - Ed.18, 90128 Palermo, Italy \\ E-mail: dominique.persanoadorno@unipa.it
}

Received 18 November 2013; accepted 4 December 2013

\begin{abstract}
Recently, electrical injection of spin polarization in n-type and p-type silicon up to room-temperature has been experimentally carried out. Despite of these promising experimental results, a comprehensive theoretical framework concerning the influence of transport conditions on the spin depolarization process in silicon structures, in a wide range of values of temperature, doping concentration and amplitude of external fields, is still in a developing stage. In this contribution we use a semiclassical multiparticle Monte Carlo approach to simulate the electron transport and spin dynamics in lightly doped n-type Si crystals and numerically calculate the spin lifetimes of drifting electrons. Spin flipping is taken into account through the Elliot-Yafet mechanism, which is dominant in group IV materials. We discuss the influence of different intravalley and intervalley phonon interactions in the spin relaxation process during the spin transport. Our findings are in good agreement with those obtained by using different theoretical approaches. Moreover, our Monte Carlo predictions, in ranges of temperature and field amplitude yet unexplored, can guide future experimental studies towards a more effective design of room-temperature silicon based spintronic-devices.
\end{abstract}

Keywords: spin relaxation process; Monte Carlo simulation; silicon; electron-phonon interactions.

PACS: 72.25.Dc, 76.60.Es, 02.70.Tt

\section{Introduction}

The process of spin relaxation in III-V semiconductor structures has been widely investigated in recent years [1-5]. Research along this line has been motivated by the possibility to develop electronic devices that perform logic operations, communication, and storage within the same material technology. However, the use of spin polarization as information carrier must face the disadvantage that each initial non-equilibrium orientation decays over time during the transport. Hence, to open the way to implementation of spin-based devices, the features of spin relaxation at relatively high temperatures, jointly with the influence of transport conditions, should be firstly fully understood and interpreted in experiment-related terms.

Recently, electrical injection of spin polarization in n-type and p-type silicon up to room temperature has been experimentally carried out [6-8]. Despite these promising experimental results, theoretical research is still incomplete and a comprehensive investigation of the influence of transport conditions on the spin depolarization process in silicon structures, in a wide range of values of temperature and amplitude of external fields, is still missing. In particular, the theoretical description of spin properties of conduction electrons in silicon bulk requires very elaborate numerical and analytical methods, the results of which are sometimes not in full agreement [9-14].

In this paper, we present for the first time the results obtained by using a semiclassical Monte Carlo (MC) approach to simulate both the electron transport and the spin dynamics. Spin relaxation is taken into account through the Elliot-Yafet mechanism [15, 16, which is dominant in group IV materials. Here, we first validate the $\mathrm{MC}$ algorithm by comparing our numerical outcomes with those provided by the most recent theoretical models. Our results are in good agreement with the findings obtained from different analytical calculations. Furthermore, this paper provides the experimental researchers with an estimate of spin lifetimes of drifting electrons in n-type Si crystals at different temperatures and under different electron transport conditions.

The paper is organized as follows: Section 2 includes some details on the silicon band structure and a brief description of the model used in our simulations for spin lifetime calculation. In Section 3 we discuss the results of the Monte Carlo code. A few concluding remarks are given in Section 4. 


\section{The model}

\subsection{Silicon model and semiclassical Monte Carlo approach}

In our Monte Carlo algorithm for the simulation of electron transport, the conduction band of silicon is represented by six equivalent $\mathrm{X}$ valleys along the $\langle 100\rangle$ directions, approximated as ellipsoids having rotational symmetry, with two effective longitudinal $m_{\mathrm{L}}$ and transverse $m_{\mathrm{T}}$ masses. Because the energy gap between the $\mathrm{X}$ and $\mathrm{L}$ valley is large $(1.05 \mathrm{eV})$, higher valleys and impact ionization are not taken into account, since for the employed electric field the electrons do not reach sufficient kinetic energies for these transitions. The code includes both the intervalley and intravalley scattering of electrons in multiple energy valleys. In particular, the scattering by acoustic phonons is taken into account, employing a deformation potential. Ionized impurity scattering is included under the Brooks-Herring approximation and the intervalley scattering is accounted for by considering six types of optical phonon, three concerning transitions between perpendicular valleys (f-type) and three among parallel valleys ( $\mathrm{g}$ type). The scattering probabilities are calculated by using the Fermi Golden Rule, and the scattering events are considered instantaneous. Scattering probabilities are assumed to be field independent; accordingly, the influence of the external fields is only indirect through the field-modified electron velocities. At this stage, we neglect the electron-electron interactions and consider electrons as being independent particles. For all the numerical simulations discussed below, we have used the parameters reported in Ref. [17, 18]. All results were obtained in $\mathrm{Si}$ with a free electron concentration of $10^{13} \mathrm{~cm}^{-3}$ (non-degenerate low-doped n-type). We assume that all donors are ionized and that free electron concentration is equal to the doping concentration.

\subsection{Spin relaxation dynamics}

In a silicon bulk, due to its inversion symmetry, the Dyakonov-Perel spin relaxation mechanism is absent and the spin depolarization process under the nondegenerate regime is mostly related to the mechanisms in which electron spins have a small chance to flip during each scattering. Spin-flip mechanisms can be classified within the Yafet and Elliott processes [15, 16. The former involves spin-dependent interactions, whereas the states are viewed as pure spin states. The Elliott processes are instead governed by spin mixing in the electron states of the conduction band due to the crystal spin-orbit coupling, whereas the interaction is spin independent. In fact, in the presence of spin-orbit interactions, the electronic Bloch states are given by a mixture of pure spin-up $|\uparrow\rangle$ and spin-down $|\downarrow\rangle$ states 15, 16):

$$
\begin{aligned}
& |\mathbf{k}, \Uparrow\rangle=\left[a_{\mathrm{k}}|\uparrow\rangle+b_{\mathrm{k}}|\downarrow\rangle\right] \mathrm{e}^{\mathrm{ikr}} \\
& |\mathbf{k}, \Downarrow\rangle=\left[a_{-\mathrm{k}}^{*}|\downarrow\rangle+b_{-\mathrm{k}}^{*}|\uparrow\rangle\right] \mathrm{e}^{\mathrm{ikr}},
\end{aligned}
$$

with the lattice momentum $\mathbf{k}$, effective spins $\Uparrow$, $\Downarrow$ and the spin-mixing parameter $\left|b_{k}\right|^{2}$. Both states have the same energy. For conduction electrons in silicon, spinflip scattering is described by a matrix element that depends on the initial and final state wave vectors as well as on the spin orientation having the form of

$$
\left\langle\mathbf{k}_{2}, \Downarrow\left|H_{\mathrm{SF}}\right| \mathbf{k}_{1}, \Uparrow\right\rangle,
$$

where $H_{\mathrm{SF}}$ is a given spin-flip mechanism. The ElliotYafet spin lifetime $\tau_{\mathrm{s}}$ can be related to the momentum relaxation time $\tau_{\mathrm{m}}$ by the following expression [15, 19. 20]:

$$
\tau_{\mathrm{s}}=\frac{\tau_{\mathrm{m}}}{4\left\langle b^{2}\right\rangle},
$$

where $\left\langle b^{2}\right\rangle$ is the Fermi surface average of the spinmixing parameter. However, this relationship is not very accurate in silicon. In fact, it has been pointed out that (i) the spin-mixing parameter $\left|b_{k}\right|^{2}$ varies in a broad range of values, depending on the value of the momentum $\mathbf{k}[19,20$, and (ii) the scattering mechanisms dominating the relaxation of the momentum are different from those leading to spin relaxation [10, 11].

The correct way to elucidate the spin relaxation mechanisms is by calculating the explicit form of the spin-flip electron-phonon matrix elements for the conduction states. Different theoretical approaches have addressed the calculation of both the spin mixing probabilities and the matrix element expressions for all the phonon-induced spin-flip transitions in the conduction band. Among these, for example, the pseudopotential model reproducing spin-orbit splittings of the relevant electronic states [ 9,10$]$, the group theory, the k•p perturbation method, the rigid-ion model [11], the parameter-free first-principles method and the density functional perturbation theory [12], the adiabatic band charge models, and the tight-binding models [13]. Starting from the detailed knowledge of a specific electron distribution, they calculate the relaxation rate $\tau_{\mathrm{i}}$ for each different scattering mechanism. If several independent mechanisms of depolarization are present, the total spin relaxation time $\tau_{\mathrm{s}}$ can be calculated by

$$
\frac{1}{\tau_{\mathrm{s}}}=\sum_{\mathrm{i}} \frac{1}{\tau_{\mathrm{i}}}
$$


Short relaxation times are most important, while the longer ones can be neglected [21].

The approach we use here is different: our MC code incorporates the electron spin dynamics by taking into account the spin mixing probabilities and the phononassisted spin-flip transitions. We consider that the rates of transitions from the $|\Uparrow\rangle$ to the $|\Downarrow\rangle$ states are proportional to the square of the matrix elements of electronlattice interaction, which causes a spin flip [15]. In particular, differently from the seminal work of Yafet [16], we take into account both intravalley and intervalley scattering. The spin mixing probability is of the order of $10^{-6}$ and depends on the value of $\mathbf{k}$ [9, 10]. In particular, more energetic electrons strongly drive the average spin relaxation. In our simplified model, we consider for the spin flipping only (i) the intravalley acoustic phonons and (ii) all six phonon modes of the intervalley f-processes. Spin flipping due to g-processes can be neglected because its contribution is not significant in the temperature range considered $(60<T<300 \mathrm{~K})$ (see Fig. 8 in Ref. [11]). More details and the explicit calculation of the spin-flip matrix elements utilized in our code can be found in Refs. [10, 11].

The dependence of spin relaxation times on temperature and/or electric field amplitude has been investigated by simulating the dynamics of $5 \cdot 10^{3}$ electrons initially polarized $(P=1)$ along the $x$ axis of the crystal at the injection plane. We calculate the polarization $P$ as a function of time by averaging over the ensemble of electrons. The spin lifetime $\tau_{\mathrm{s}}$ corresponds to the time necessary to achieve a reduction of the initial spin polarization by a factor $1 / \mathrm{e}$.

\section{Results and discussion}

First, we calculated the contribution of acoustic (AC) and optical (OP) phonon scattering to the spin relaxation process separately, in absence of the applied electric field. The spin relaxation time $\tau_{s}$ as a function of the crystal temperature is shown in Fig. 1, where the contributions of AC phonon (upper panel) or OP phonon (lower panel) scattering mechanisms are considered. In each panel, our numerical findings are compared with the results provided by recent theoretical approaches. The agreement with the analytical trends is satisfactory in the whole range of investigated lattice temperatures. Moreover, a comparison between the acoustic and optical phonon contribution to the spin relaxation shows that the AC phonon scattering is dominant in a low temperature range $(T<200 \mathrm{~K})$, while OP phononassisted spin-flip transitions cannot be longer neglected at higher temperatures. This is consistent with the circumstance that at smaller energies (lower temperatures) long-wavelength acoustic phonon modes are larger populated, whereas the growing up of the phonon population with the temperature for optical modes is faster than for acoustic modes.

In Fig. 2, the electron spin total lifetime computed in our simulations is compared, as a function of temperature, with recent theoretical and analytical findings. Despite that our MC code does not still take into account the scattering of electrons with g-phonons, the interactions with impurities and uses approximated relations for the spin-flip rates, a good agreement with the analytical theories in the absence of applied electric fields strengthens the reliability of our MC algorithm.
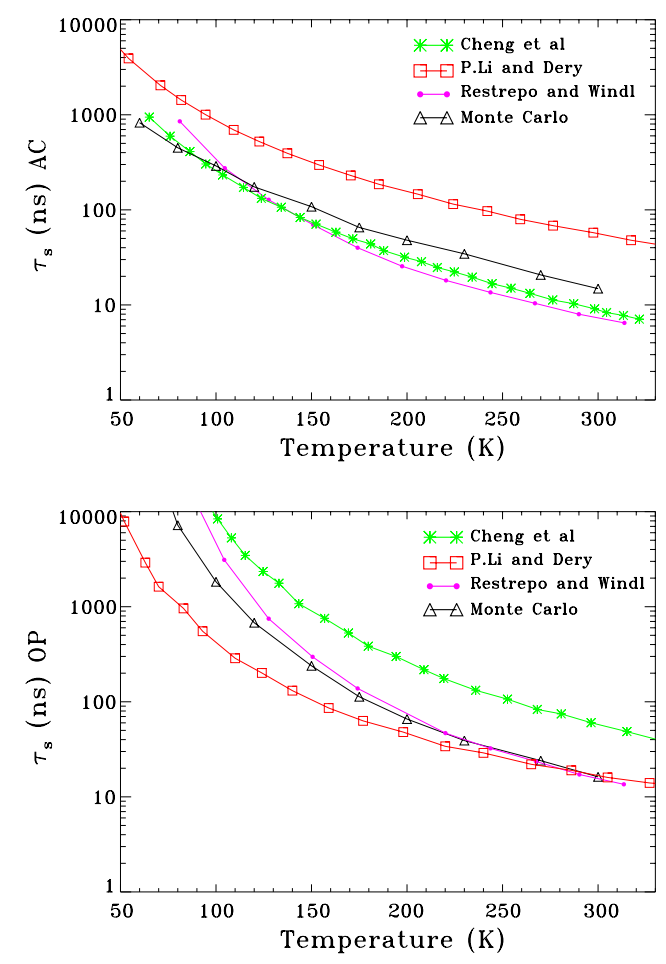

Fig. 1. Comparison between the MC-computed contributions to the spin relaxation process of acoustic (AC) phonon (upper panel) and optical (OP) phonon (lower panel) scattering and the results from recent theoretical approaches, as a function of temperature.

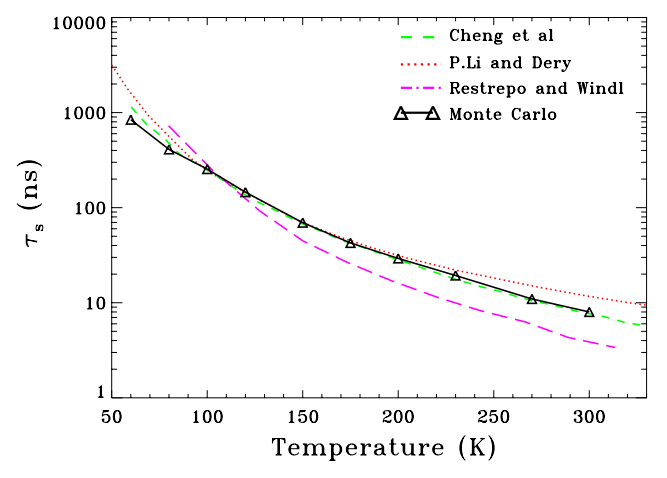

Fig. 2. Comparison between our MC numerical total spin lifetime and the results from recent theoretical approaches, as a function of temperature. 
Figure 3 shows the calculated spin lifetimes in the presence of an electric field applied along the $\mathrm{x}$-axis of the crystal at three different temperatures, namely $T=90,120$, and $300 \mathrm{~K}$.

The presence of the driving electric field significantly affects the electron momentum distribution that deviates from the equilibrium condition even at low temperatures. This fact is responsible for the enhancement of intervalley scattering and explains a rapid drop of spin lifetimes observed at electric fields higher than $3 \mathrm{kV} / \mathrm{cm}$. At room temperature, where up today no experimental data are yet available, our calculation predicts spin lifetimes of the order of $8 \mathrm{~ns}$ up to field amplitudes of $\sim 5 \mathrm{kV} /$ $\mathrm{cm}$. When the electric field amplitude is maximum $(E=20 \mathrm{kV} / \mathrm{cm})$, the effect of temperature becomes nearly negligible; all the three curves collapse and the spin relaxation times take values ranging between 1 and 2 ns.

\section{Conclusion}

Preliminary results of MC calculations of the spin lifetime for conduction electrons drifting in a silicon bulk are reported. We show how spin lifetimes change in a wide range of temperature and electric field amplitude, also where experimental and/or analytical data are not yet available. From this point of view, our Monte Carlo simulations can guide experimental studies and be very useful in designing efficient silicon-based spintronic devices.

\section{Acknowledgements}

This work was partially supported by MIUR and CNISM.

\section{References}

[1] J.M. Kikkawa and D.D. Awschalom, Phys. Rev. Lett. 80, 4313-4316 (1998).

[2] J. Fabian, A. Matos-Abiague, C. Ertier, P. Stano, and I. Zutic, Acta Phys. Slovaca 57, 565-907 (2007).

[3] M.W. Wu, J.H. Jiang, and M.Q. Weng, Phys. Rep. 493, 61-236 (2010).

[4] S. Spezia, D. Persano Adorno, N. Pizzolato, and B. Spagnolo, J. Stat. Mech. Theor. Exp. 11, P11033 (2010).

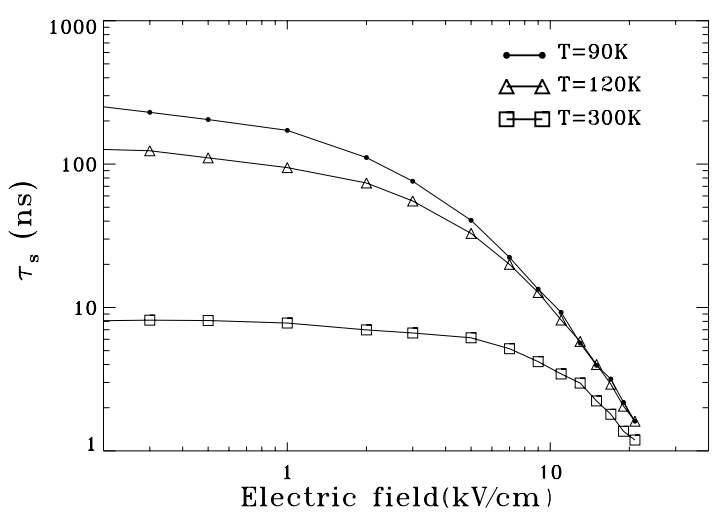

Fig. 3. Electron spin lifetime as a function of the applied electric field at three different values of temperature.

[5] S. Spezia, D. Persano Adorno, N. Pizzolato, and B. Spagnolo, Acta Phys. Pol. B 41, 1171-1180 (2010).

[6] B.T. Jonker, G. Kioseoglou, A.T. Hanbicki, C.H. Li, and P.E. Thompson, Nat. Phys. 3, 542-546 (2007).

[7] I. Appelbaum, B.Q. Huang, and D.J. Monsma, Nature 447, 295-298 (2007).

[8] S.P. Dash, S. Sharma, R.S. Patel, M.P. de Jong, and R. Jansen, Nature 462, 491-494 (2009).

[9] J.L. Cheng, M.W. Wu, and J. Fabian, Phys. Rev. Lett. 104, 016601-4 (2010).

[10] P. Li and H. Dery, Phys. Rev. Lett. 107, 107203-5 (2011).

[11]Y. Song and H. Dery, Phys. Rev. B 86, 085201-28 (2012).

[12] O.D. Restrepo and W. Windl, Phys. Rev. Lett. 109, 166604-5 (2012).

[13] J.M. Tang, B.T. Collins, and M.E. Flattè, Phys. Rev. B 85, 045202-7 (2012).

[14]J. Li, L. Qing, H. Dery, and I. Appelbaum, Phys. Rev. Lett. 108, 157201-5 (2012).

[15]R.J. Elliott, Phys. Rev. 96, 266-279 (1954).

[16]Y. Yafet, in: Solid State Physics, eds. F. Seitz and D. Turnbull, vol. 14 (Academic Press, New York, 1963).

[17]D. Persano Adorno, M. Zarcone, and G. Ferrante, Laser Phys. 11, 291-295 (2001).

[18]D. Persano Adorno, M. Zarcone, and G. Ferrante Laser Phys. 13, 270-274 (2003).

[19]J. Fabian and S. Das Sarma, Phys. Rev. Lett. 81, 5624-5627 (1998).

[20]J. Fabian and S. Das Sarma, Phys. Rev. Lett. 83, 1211-1214 (1999).

[21] A.W. Overhauser, Phys. Rev. 89, 689-700 (1953).

\title{
LAIDUMO ELEKTRONŲ SUKINIŲ RELAKSACIJOS SILICYJE MODELIAVIMAS MONTE KARLO METODU
}

\author{
D. Persano Adorno, C. Graceffa, N. Pizzolato, M.A. Lodato
}

Palermo universitetas, Palermas, Italija 